Journal for ImmunoTherapy of Cancer

\title{
T cell engaging bispecific antibodies targeting CD33 IgV and IgC domains for the treatment of acute myeloid leukemia
}

\author{
Sayed Shahabuddin Hoseini, ${ }^{1,2}$ Mallika Vadlamudi, ${ }^{2}$ Madelyn Espinosa-Cotton (1) , \\ Hoa Tran, ${ }^{1}$ Yi Feng, ${ }^{1}$ Hong-fen Guo, ${ }^{1}$ Hong Xu, ${ }^{1}$ Irene Cheung, ${ }^{1}$ \\ Nai-Kong $\vee$ Cheung (D) ${ }^{1}$
}

To cite: Hoseini SS, Vadlamudi M, EspinosaCotton $\mathrm{M}$, et al. T cell engaging bispecific antibodies targeting CD33 IgV and IgC domains for the treatment of acute myeloid leukemia. Journal for ImmunoTherapy of Cancer 2021;9:e002509. doi:10.1136/ jitc-2021-002509

Accepted 18 April 2021
Check for updates

(C) Author(s) (or their employer(s)) 2021. Re-use permitted under CC BY-NC. No commercial re-use. See rights and permissions. Published by BMJ.

${ }^{1}$ Pediatrics, Memorial Sloan Kettering Cancer Center, New York, New York, USA

${ }^{2}$ Ymabs Therapeutics, Nutley, New Jersey, USA

Correspondence to Dr Nai-Kong V Cheung; cheungn@mskcc.org

\begin{abstract}
Background Acute myeloid leukemia (AML) remains one of the most challenging hematological malignancies. Despite progress in therapeutics, majority of patients succumb to this neoplasm. CD33 is a proven therapeutic target, given its expression on most AML cells. Almost all anti-CD33 antibodies target the membrane distal immunoglobulin V (lgV) domain of the CD33 extracellular domain.

Methods In this manuscript, we present data on three bispecific antibodies (BsAbs) against the CD33 IgV and membrane proximal immunoglobulin $\mathrm{C}(\mathrm{lgC})$ domains. We use in vitro binding and cytotoxicity assays to show the effect of these BsAbs on AML cell lines. We also use immunodeficient mice-bearing leukemias from cell lines and patient-derived xenografts to show the effect of these BsAbs in vivo.

Results In vitro, the IgV-targeting BsAb had higher binding to AML cell lines using flow cytometry and delivered more potent cytotoxicity in T-cell-dependent cytotoxicity assays; importantly, the IgC domain-targeting outperformed the IgV domain-targeting BsAb in medullary and extramedullary leukemia animal models.

Conclusions These data support further clinical development of this BsAb for first-in-human phase I clinical trial.
\end{abstract}

\section{INTRODUCTION}

Leukemia is the most common cancer in children. Despite advances in the treatment of acute lymphoblastic leukemia, patients with acute myeloid leukemia (AML) have a much worse prognosis, with a 3-year overall survival rate of about $20 \% .^{1}$ The outcome of adult patients with AML is even poorer, with only $6 \%$ of patients older than 65 surviving 2 years after diagnosis. ${ }^{2}$ There is an obvious unmet need for better AML therapies.

CD33, a member of the sialic acid-binding immunoglobulin-like (Ig-like) lectin family, is expressed on most AML. The CD33 extracellular domain is composed of a membrane distal immunoglobulin $\mathrm{V}(\mathrm{IgV})$ and $\mathrm{a}$ membrane proximal immunoglobulin $\mathrm{C}$ (IgC) domain. Current anti-CD33 antibodies in clinical trials, including the only Food and Drug Administration-approved antibody gemtuzumab ozogamicin (GO), target the $\mathrm{IgV}$ domain.

Single nucleotide polymorphism (SNP) rs12459419 $\mathrm{C}>\mathrm{T}$ in exon 2 of CD33 antigen eliminates the expression of the $\mathrm{IgV}$ domain and reduces the expression of the full-length protein. This SNP, which occurs in almost $50 \%$ of AML cases, renders the patients unresponsive to GO, a drug that targets the $\mathrm{IgV}$ domain. ${ }^{3}$ To tackle this splice variant heterogeneity, we generated $\mathrm{T}$ cell engaging bispecific antibodies (BsAbs) targeting the $\mathrm{IgV}$ and IgC domains and compare their function in vitro and potency in vitro in xenograft models of human AML.

\section{MATERIALS AND METHODS Antibody humanization}

The murine M195 anti-CD33 antibody was humanized as explained earlier. ${ }^{4}$ The murine My96 anti-CD33 was humanized by grafting the heavy chain complementarity determining region (CDR) sequences onto the human framework IGHV1-46*01 and IGHJ $4 * 01$ and the light chain CDR sequences onto the human framework IGKV4- $1 * 01$ and IGKJ $4 * 01$. The murine HIM3-4 anti-CD33 was humanized by grafting the heavy chain CDR sequences onto the human framework IGHV1-2*02 and IGHJ4*01 and the light chain CDR sequences onto the human framework IGKV1-33*01 and IGKJ2*01.

The CD3xCD33 BsAbs were designed using heavy chain variable region fragment/ light chain variable region fragment $(\mathrm{VH} /$ VL) domains from huM195, huMy96, and 


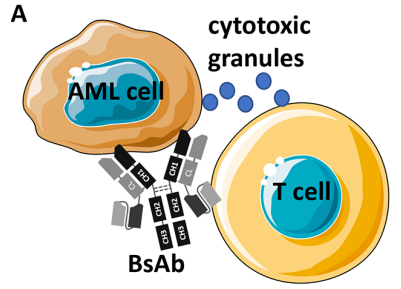

D

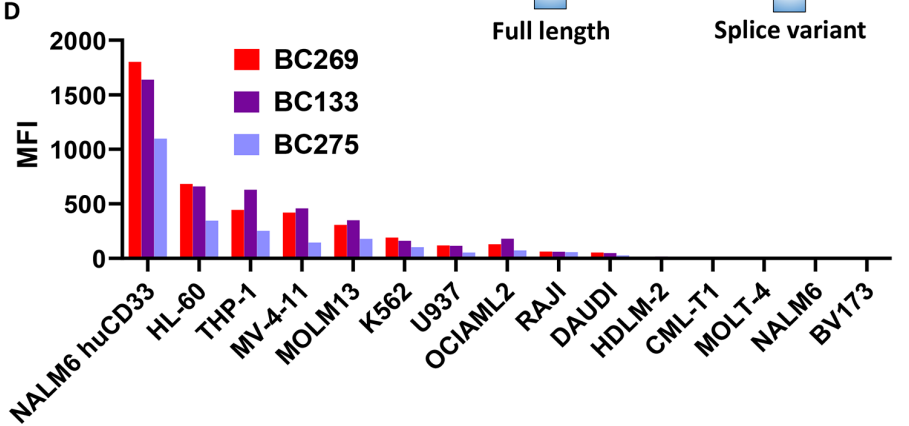

B

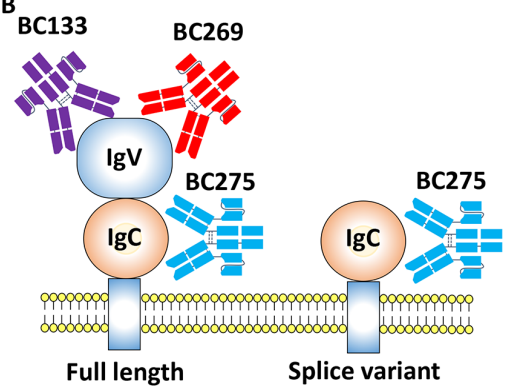

C

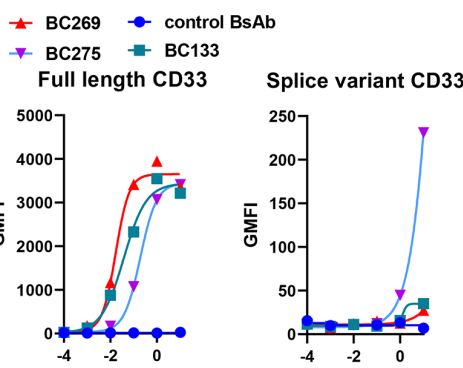

Figure 1 Bispecific antibodies (BsAbs) binding to the $\lg V$ or the $\lg C$ domains of CD33. (A, B) Schematic of the three CD3xCD33 T cell engaging BsAbs against the IgV (BC133 and BC269) and IgC (BC275) extracellular domains of CD33. (C) 293T cells were transiently transfected with DNA plasmids containing the gene for full-length human CD33 or the splice variant and stained with BC133, BC269, BC275, and an irrelevant control antibody. (D) Several CD33(+) and CD33(-) cell lines were stained with BC133, BC269 and BC275. A secondary anti-human Fc fluorochrome-conjugated antibody was used to stain the cells. AML, acute myeloid leukemia; GMFI, geometric mean fluorescence intensity; IgC, immunoglobulin C; IgV, immunoglobulin $\mathrm{V}$; MFI, mean fluorescence intensity.

huHIM3-4 antibodies and huOKT3 scFv fused to the $\mathrm{C}$ terminus of the light chain of a human IgG1 as previously described and were named BC133, BC269, and BC275, respectively. ${ }^{5}$ The N297A and K322A mutations were generated to eliminate Fc glycosylation and complement binding, respectively. ${ }^{6}$

\section{Surface plasmon resonance}

Human CD33 (Sino Biological, Cat \# 12238-H08H) was immobilized on a CM5 chip. Serial twofold dilutions of the BsAbs (starting at $100 \mathrm{nM}$ ) were flowed over the chip using a Biacore T200 system. Binding kinetics were measured at $37^{\circ} \mathrm{C}$. The sensorgrams were fitted with a two-state binding model.

\section{Cytotoxicity assay $\left({ }^{51} \mathrm{Cr}\right.$ release assay)}

Leukemia cells were cultured in RPMI 1640 medium (Cellgro) supplemented with $10 \%$ heat-inactivated fetal bovine serum (FBS) (Life Technologies) at $37^{\circ} \mathrm{C}$ in a $5 \%$ $\mathrm{CO}_{2}$ humidified incubator. Standard chromium release cytotoxicity assays were performed using activated human T cells as described before. ${ }^{4}$

\section{T cell activation, proliferation, and cytokine release}

Buffy coats were purchased from the New York Blood Center. Peripheral blood mononuclear cells (PBMCs) were isolated from buffy coats using the lymphocyte separation medium (Corning, Cat \# 25-072-CV). T cells were purified from PBMCs using the EasySep Human T Cell Isolation Kit (Stem Cell Technologies, Cat \# 17951). For $\mathrm{T}$ cell proliferation assays, carboxyfluorescein succinimidyl ester (CFSE)-labeled (Thermo Scientific, Cat \# C34554) effector cells were mixed with AML cells with an E:T ratio of 10 with different concentrations of BC275 for 4 days before being assessed by flow cytometry (Attune, Thermo Scientific). The percentage of cells with diluted CFSE signal was used as the proliferation readout. For $\mathrm{T}$ cell activation and proliferation assays, effector cells were mixed with AML cells (E:T of 10) and dilutions of BC275 for 1 day (early activation marker CD69 and cytokine release assay) or 4 days (late activation marker CD25). For the activation assays, cells were assessed using a flow cytometer using anti-human

Table 1 Binding kinetics of CD3xCD33 bispecific antibodies to human CD33

\begin{tabular}{llllll}
\hline & ka (1/Ms) & kd (1/s) & ka2 (1/s) & kd2 (1/s) & KD (M) \\
\hline BC269 & $7.84 \mathrm{E}+05$ & $6.58 \mathrm{E}-04$ & $3.12 \mathrm{E}-03$ & $4.30 \mathrm{E}-04$ & $1.02 \mathrm{E}-10$ \\
BC133 & $9.85 \mathrm{E}+05$ & $7.97 \mathrm{E}-03$ & $2.06 \mathrm{E}-03$ & $9.02 \mathrm{E}-04$ & $2.47 \mathrm{E}-09$ \\
BC275 & $3.37 \mathrm{E}+05$ & $1.36 \mathrm{E}-03$ & $3.72 \mathrm{E}-03$ & $7.13 \mathrm{E}-04$ & $6.49 \mathrm{E}-10$ \\
\hline
\end{tabular}

ka2, equilibrium association constant 2; ka, equilibrium association constant; kd, equilibrium dissociation constant; kd2, equilibrium dissocation constant 2; KD (M), equilibrium dissociaiton constant molarity. 
Table 2 Humanness of the CD33 binding moieties of the BsAbs

\begin{tabular}{llll}
\hline & BC133 & BC269 & BC275 \\
\hline VL humanness & 76.6 & 91 & 88.3 \\
VH humanness & 76.5 & 86 & 85.7 \\
\hline
\end{tabular}

$\mathrm{VH}$, heavy chain variable region fragment; VL, light chain variable region fragment.

CD69 (Biolegend, Cat \# 310906), CD25 (Biolegend, Cat \# 356104), CD4 (Biolegend, Cat \# 344612), and CD8 (Biolegend, Cat \# 344722). The cytokine concentration in the supernatant (after 24 hours of culture) was measured using the LegendPlex Human Th1 Panel (Biolegend, Cat \# 741035).

\section{Cell lines and analysis by flow cytometry}

The following cell lines were used in this paper: Kasumi-1 (ATCC Cat \# CRL-2724, RRID:CVCL_0589), MOLM-13 (DSMZ Cat \# ACC-554, RRID:CVCL_2119), SKM1 (DSMZ Cat \# ACC 547, RRID:CVCL_0098), HL60 (ATCG Cat \# CCL-24), THP1 (ATCC Cat \# TIB-202), MV4-11
(ATCC Cat \# CRL-9591), K562 (ATCC Cat \# CCL-243), U937 (ATCC Cat \# CRL-1593.2), OCIAML2 (DSMZ Cat \# ACC 99), Raji (ATCC Cat \# CCL-86), Daudi (ATCC Cat \# CCL-213), HDLM-2 (DSMZ Cat \# ACC 17), CML-T1 (DSMZ Cat \# ACC 7), MOLT4 (ATCC Cat \# CRL-1582), NALM6 (gift from Dr David Scheinberg's Lab), BV173 (DSMZ Cat \# ACC 20). The cells were cultured in RPMI 1640 (Gibco) supplemented with 10\%-20\% FBS (Gibco) at $37^{\circ} \mathrm{C}$ in a $5 \% \mathrm{CO}_{2}$ humidified incubator. Cryopreserved bone marrow (BM) and cord blood (CB)-derived CD34(+) hematopoietic stem and progenitor cells (HSPCs) were purchased from ZenBio (Cat \# SER-BMCD34-F and SERCD34-1F, respectively).

The human CD33 plasmids, gifts from Trinidad Hernández-Caselles of the IMIB-University of Murcia, ${ }^{7}$ were cloned into lentiviral vectors. NALM6 cells were transduced with a lentiviral vector expressing the human CD33 gene. 293T cells (Thermo Fisher Scientific) were transiently transfected with DNA plasmids containing the full-length or splice variant (truncated) human CD33 genes. Goat anti-human antibody (Southern Biotech, Cat \# 2040-09) was used to detect humanized antibodies.

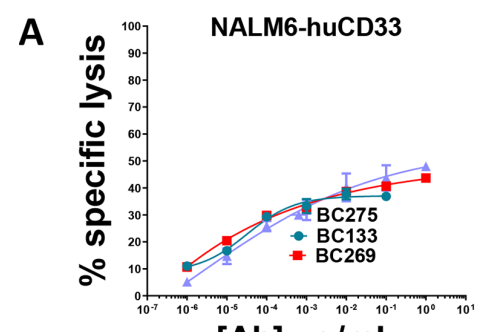

[Ab] $\mathrm{ug} / \mathrm{mL}$

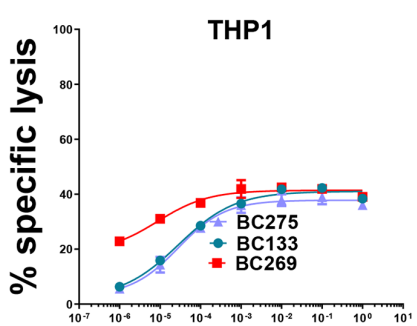

[Ab] $\mathrm{ug} / \mathrm{mL}$

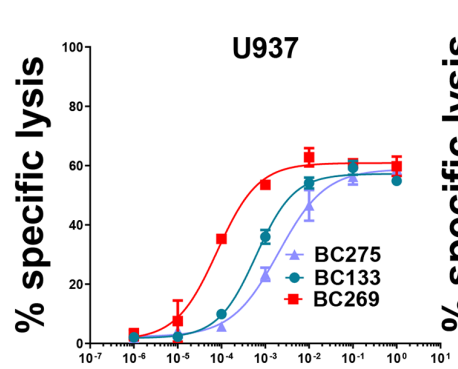

[Ab] $\mathrm{ug} / \mathrm{mL}$

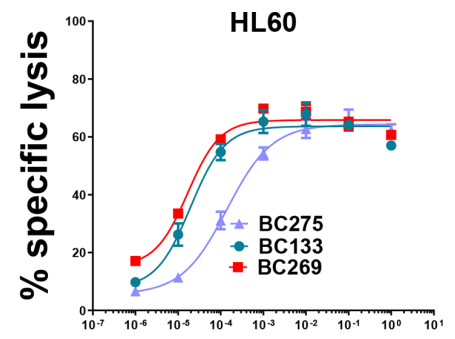

[Ab] ug/mL

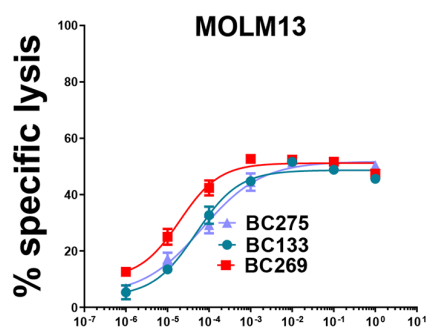

$[\mathrm{Ab}] \mathrm{ug} / \mathrm{mL}$

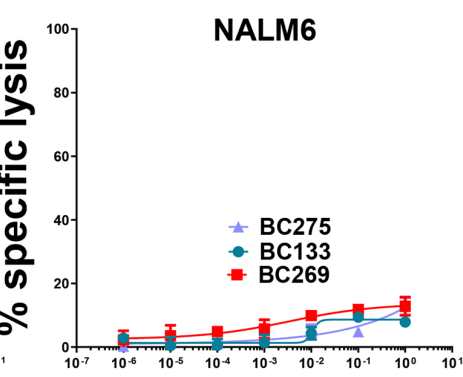

[Ab] ug/mL

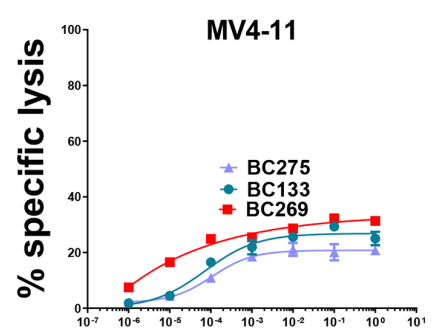

$[\mathrm{Ab}] \mathrm{ug} / \mathrm{mL}$

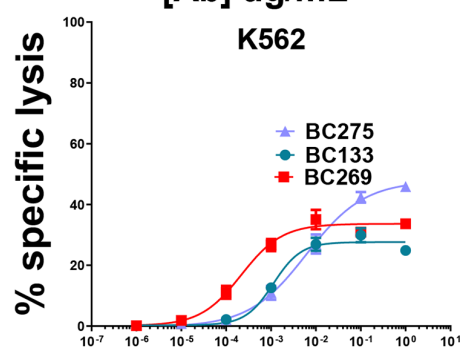

[Ab] ug/mL

B

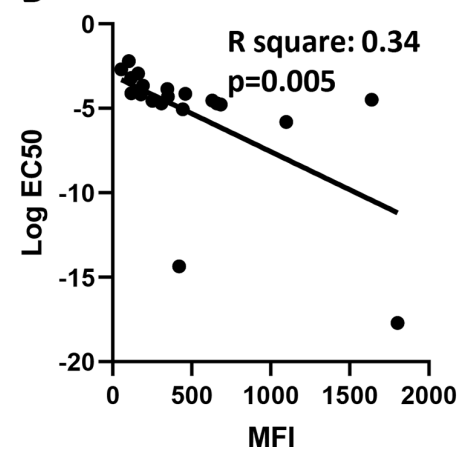

Figure 2 T-cell-mediated cytotoxicity of the anti-CD33 bispecific antibodies (BsAbs) in vitro. (A) Activated T cells were cultured with ${ }^{51} \mathrm{Cr}$-labeled MOLM13 cells $(\mathrm{E}: \mathrm{T}$ ratio $=10)$ in the presence of different doses of the BsAbs for 4 hours. Release of ${ }^{51} \mathrm{Cr}$ was measured as an indicator of cytotoxicity. (B) The correlation between mean fluorescence intensity (MFI) measured by flow cytometry and the log EC50 of the cytotoxicity assays is plotted (R square: $0.34 ; p=0.005)$. This plot includes EC50s of all BsAbs from all the cell lines shown in this figure. 


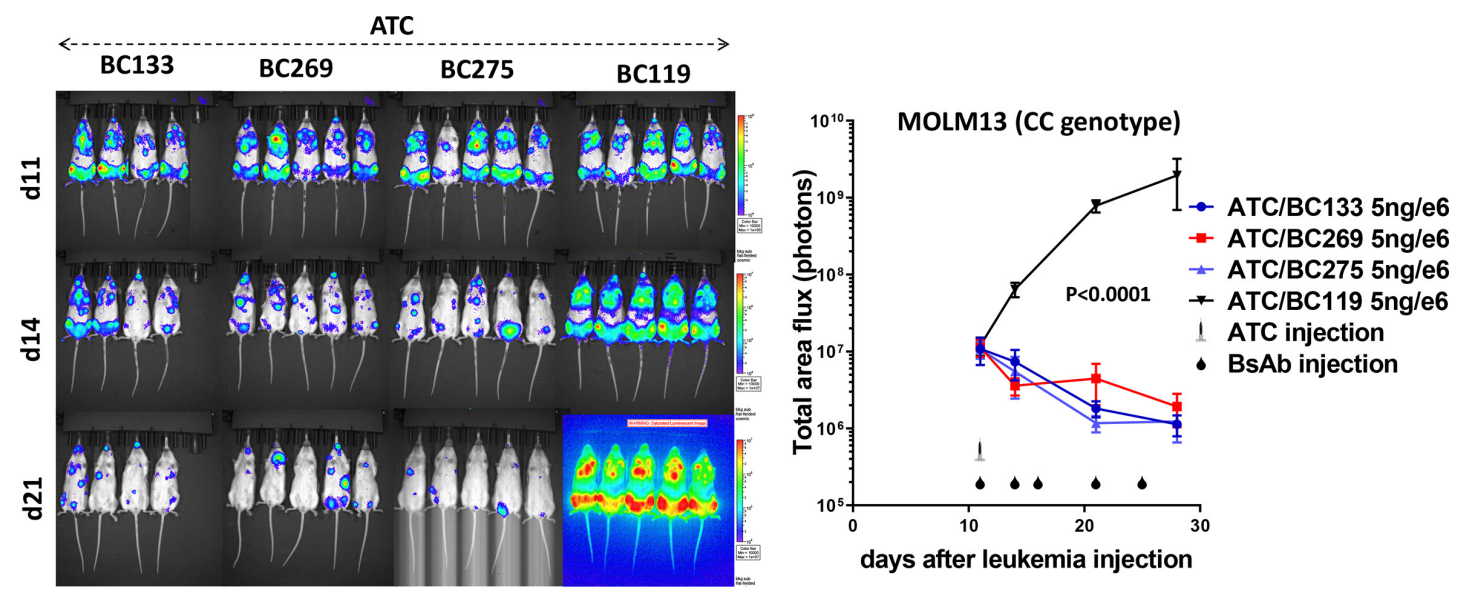

Figure 3 Low dose of the CD33-specific bispecific antibodies (BsAbs) redirects polyclonal T cells to treat acute myeloid leukemia (AML) xenografts in vivo. Immunodeficient NOD.Cg-Prkdc ${ }^{\text {scid }} I \mathrm{LL}-2 \mathrm{R}-\gamma^{\mathrm{tm}}{ }^{1} \mathrm{Wj} / \mathrm{SzJ}$ (NSG) mice were intravenously inoculated with CD33(+) MOLM13-luciferase human AML xenografts $\left(10^{6}\right.$ cells). Therapy was started after 11 days. Mice received a single injection of $5 \times 10^{6}$ activated T cells with $5 \mathrm{ng} / 10^{6} \mathrm{~T}$ cells of the BsAbs. In the control group, mice received T cells plus BC119 (CD3×GD2 BsAb). Injection of the BsAbs alone was continued for four more doses. Leukemia growth was monitored using the IVIS bioluminescent imager. Area under the curve of the log total flux was compared between groups using analysis of variance and multiple comparison analysis.

Directly conjugated antibodies were purchased from Biolegend. Flow cytometry was carried out using either the FACSCalibur (BD Biosciences) or Attune Nxt (Thermo Fisher Scientific) flow cytometer and analyzed with FlowJo software (FlowJo, LLC, Ashland, Oregon, USA).

\section{In vivo studies}

All mouse experiments were performed in compliance with the Institutional Animal Care and Use Committee guidelines. The immunodeficient BALB- $\operatorname{Rag}^{-/-} \mathrm{IL}-2 \mathrm{R}-$ $\gamma \mathrm{c}-\mathrm{KO} \quad$ (BRG) and NOD.Cg-Prkdc ${ }^{\mathrm{scid}}$ IL-2R- $\gamma^{\mathrm{tm} 1 \mathrm{Wjl}} / \mathrm{Sz} \mathrm{J}$ (NSG) mice were purchased from Taconic (Hudson, New York, USA), Jackson Laboratory (Bar Harbor, Maine, USA), or maintained at Memorial Sloan Kettering Cancer Center and fed with sulfatrim. For in vivo xenograft experiments, 6 -week to 10 -week-old mice were inoculated with leukemic cells (subcutaneously in Matrigel (BD Biosciences) or intravenously). Activated $\mathrm{T}$ cells were incubated
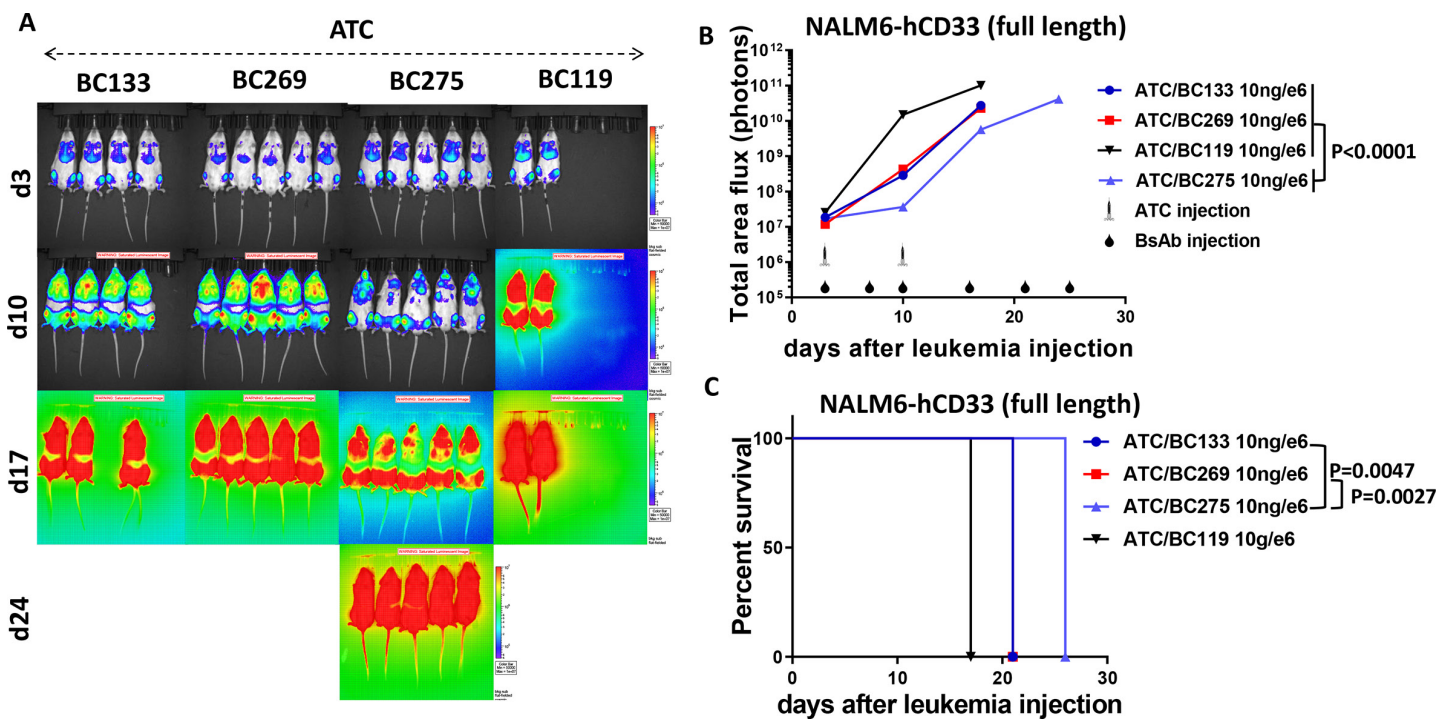

Figure 4 BC275 compared with BC133 or BC269 mediates stronger T cell killing against human CD33-transduced leukemia xenografts in vivo. Immunodeficient NOD.Cg-Prkdc scid IL-2R- $\gamma^{\mathrm{tm} 1 \mathrm{Wjl}} / \mathrm{SzJ}$ (NSG) mice were intravenously inoculated with CD33transduced NALM6-luciferase human leukemia xenografts $\left(0.5 \times 10^{6}\right.$ cells). Therapy was started after 3 days. Mice received

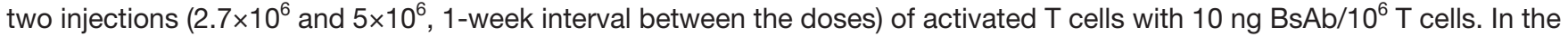
control group, mice received T cells plus BC119 (CD3×GD2 BsAb). Injection of the BsAbs alone was continued for four more doses. (A, B) Leukemia growth was monitored using the IVIS bioluminescent imager. To support T cell engraftment, all mice received two subcutaneous injections of interleukin-2 (1000 IU) during the first week of treatment. Area under the curve of the log total flux until day 17 was compared between groups using analysis of variance and multiple comparison analysis. (C) Survival analysis was done by log-rank test. 
A

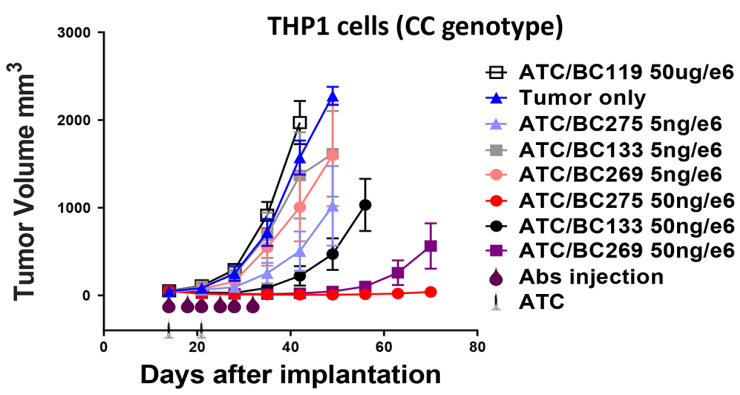

B

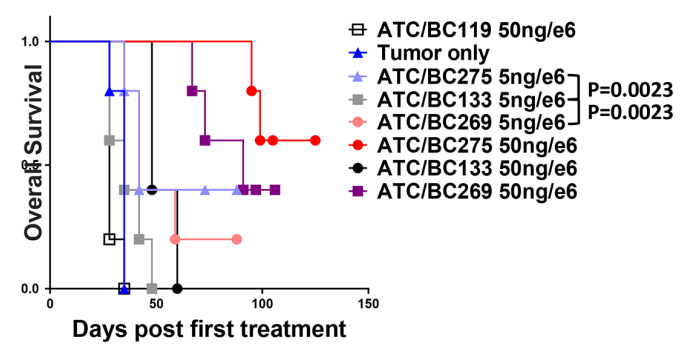

C

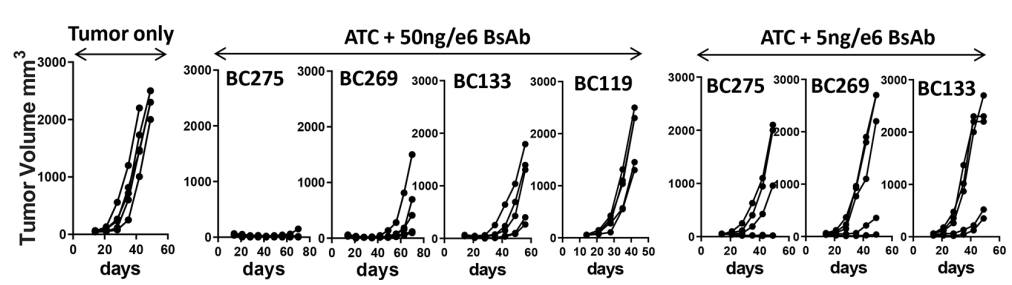

Figure 5 BC275 outperforms BC133 or BC269 against extramedullary human acute myeloid leukemia (AML) xenografts in vivo. Immunodeficient BALB-Rag $2^{-/}{ }^{I L}-2 \mathrm{R}-\gamma \mathrm{\gamma}-\mathrm{KO}$ (BRG) mice were subcutaneously inoculated with THP1 human leukemia xenografts $\left(3 \times 10^{6}\right.$ cells). Therapy was started after 14 days. Mice received two injections $\left(10^{7}\right.$ each) of activated T cells with 5-50 ng bispecific antibodies (BsAbs) $/ 10^{6}$ T cells. In the control group, mice received T cells plus BC119 (CD3×GD2 BsAb). Injection of the BsAbs alone was continued for four more doses. (A) Growth of leukemia was measured by a caliper. (B) Overall survival is shown. Survival analysis was done by log-rank test. (C) Tumor size of each individual mouse is shown. To support T cell engraftment, all mice received subcutaneous injections of interleukin-2 (1000 IU).

with the BsAbs for 20 min and injected intravenously. In addition, 1000 IU of interleukin-2 (IL-2) was subcutaneously injected two times per week. Tumor growth was measured once per week by using a Peira TM900 imaging device (Peira, Turnhout, Belgium) or an IVIS bioluminescence imaging system.

\section{RESULTS}

\section{Anti-CD33xCD3 BsAbs target different CD33 extracellular} domains and bind to AML cells in vitro

We generated BsAbs engaging human $\mathrm{T}$ cells (via CD3) to AML cells (via CD33) using the IgG-L-scFv format (figure 1A) ${ }^{8}$ Two of the BsAbs target the CD33 IgV extracellular domain (BC133 based on the antiCD33 antibody lintuzumab (M195 clone) sequence and BC269 based on the anti-CD33 antibody gemtuzumab (My96 clone) sequence), while one of the BsAbs targets the CD33 IgC extracellular domain (based on the anti-CD33 antibody HIM3-4 sequence) (figure 1B). We transiently expressed two versions of human CD33 (the truncated splice variant with only the IgC domain and the full-length version with both $\mathrm{IgV}$ and $\mathrm{IgC}$ domains) in $293 \mathrm{~T}$ cells and assessed the ability of our BsAbs to bind using flow cytometry. Figure 1C shows that while BC133, BC269, and BC275 all bind the full length CD33, only BC275 binds the splice variant.

To test the specific binding of these BsAbs to myeloid leukemia, various AML and non-AML cell lines were stained with the BsAbs. As shown in figure 1D, all
BsAbs bind AML cells. Interestingly, the maximum binding of these antibodies to AML cells was higher for BC133 and BC269 when compared with BC275. To investigate the mechanism for lower BC275 binding to AML cells, affinity of the BsAb to human CD33 was measured by surface plasmon resonance (SPR). Interestingly, $\mathrm{BC} 133$ had the weakest affinity to CD33 (2 nM), while BC275 and BC269 had higher binding affinity (0.65 and $0.1 \mathrm{nM}$, respectively) (table 1$)$. These data demonstrate that the lower cell binding of BC275 in flow cytometry is not due to poorer affinity and suggest a lower epitope accessibility of CD33 IgC domain as the reason for lower antibody binding. In table 2, the humanness of the three BsAbs is shown. Both BC269 and BC275 have higher human content than BC133.

\section{Anti-CD33xCD3 BsAbs engage activated human T cells to lyse different CD33(+) AML cells in vitro}

To test the potency and specificity of the BsAbs in vitro, CD33(+) and CD33(-) leukemia cells were cultured with activated human $\mathrm{T}$ cells in the presence of decreasing concentrations of the BsAbs. All BsAbs lysed CD33(+) leukemic cells with low picomolar to high femtomolar EC50 (figure 2A), while CD33(-) NALM6 cells were unaffected. Among these three antibodies, BC269 was the most potent antibody in the in vitro cytotoxicity assays. In addition, there was a positive correlation between the MFI and the EC50 cytotoxic potency of the BsAb (figure 2B). 

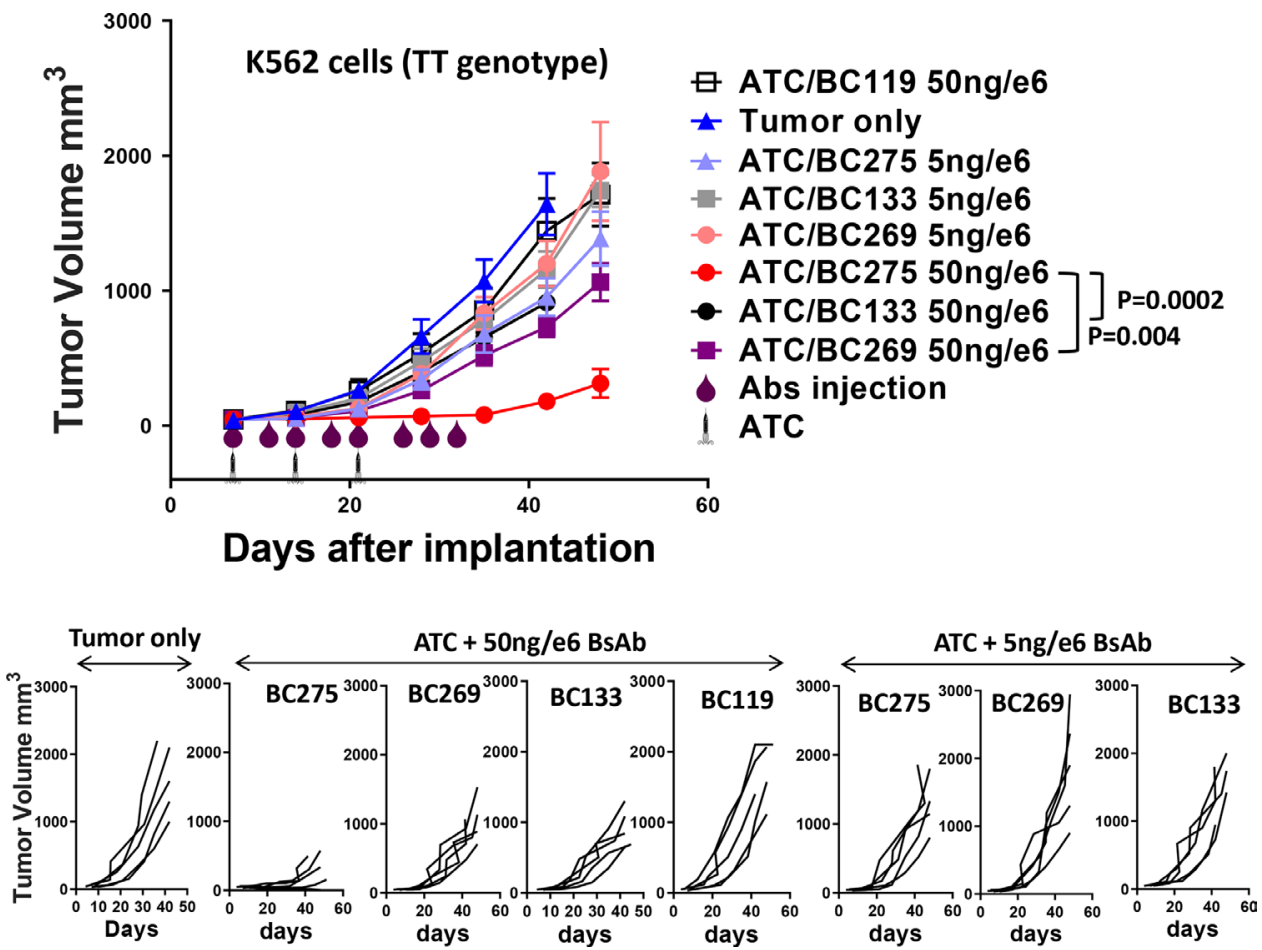

Figure 6 BC275 outperforms BC133 or BC269 against extramedullary human acute myeloid leukemia (AML) xenografts with the TT rs12459419 single nucleotide polymorphism (SNP) in vivo. Immunodeficient BALB-Rag2 ${ }^{-/}$IL-2R- $\gamma \mathrm{C}-\mathrm{KO}$ (BRG) mice were subcutaneously inoculated with THP1 human leukemia xenografts $\left(3 \times 10^{6}\right.$ cells). Therapy was started after 14 days. Mice received two injections ( $10^{7}$ each) of activated T cells with $5-50 \mathrm{ng}$ bispecific antibodies (BsAbs)/ $/ 10^{6} \mathrm{~T}$ cells. In the control group, mice received T cells plus BC119 (CD3×GD2 BsAb). Injection of the BsAbs alone was continued for four more doses. Growth of leukemia was measured using a caliper. To support T cell engraftment, all mice received subcutaneous injections of interleukin-2 (1000 IU). Area under the curve of the log total flux until day 42 was compared between groups with $50 \mathrm{ng} / \mathrm{e} 6 \mathrm{BsAb}$ using analysis of variance and multiple comparison analysis.

\section{BC275 outperforms the other BsAbs in human xenograft mouse models}

In vivo potency of these BsAbs were next tested in AML xenografted mice. In the first set of experiments, NSG mice were intravenously engrafted with MOLM13 AML xenografts (carrying the CC SNP genotype of CD33). Treatment consisted of one dose of $\mathrm{T}$ cells and multiple doses of BsAbs. All BsAbs were able to control leukemia growth in contrast with control $\mathrm{BsAb}$ (figure 3). To differentiate the potency of the antibodies further, fast-growing NALM6-luciferase leukemia cells that were transduced with the human CD33 gene were engrafted in NSG mice. A low dose of the BsAbs (10 ng/ $10^{6} \mathrm{~T}$ cells) plus a low dose of $\mathrm{T}$ cells were used for treatment. BC275 slowed down leukemia growth $(\mathrm{p}<0.0001)$ and extended overall survival more than BC133 $(\mathrm{p}=0.0047)$ or BC269 $(\mathrm{p}=0.0027)$ (figure 4).

\section{BC275 has superior potency against human AML xenografts in extramedullary leukemia models}

Treatment of extramedullary leukemia can be a clinical challenge. To simulate this type of cancer, immunodeficient BRG mice were engrafted subcutaneously with THP1-human AML xenografts cells (CC SNP genotype). Treatment consisted of two doses of $10^{7}$ activated $T$ cells with decreasing doses of the BsAbs.
Although all three BsAbs could delay tumor growth, BC275 appeared to extend survival of the mice more, although the difference between BC275 and BC269 did not reach statistical significance (figure 5). Furthermore, $60 \%$ of mice treated with BC269 had tumor recurrence $\left(>150 \mathrm{~mm}^{3}\right)$, while none of the mice treated with BC275 experienced tumor recurrence by day 70 when the mice had to be sacrificed because of graft versus host disease. To compare the BsAbs against cell lines with the TT SNP genotype, which have a frequency of about $10 \%$ of AML cases $^{3}$ and have lower expression of the full-length CD33, K562 cells (TT SNP genotype) were engrafted subcutaneously into BRG mice. The mice were treated with weekly injections of $10^{7} \mathrm{~T}$ cells for 3 weeks in addition to injections of the BsAbs. Subcutaneous IL-2 weekly was necessary to maintain $T$ cell survival in the BRG mouse strain. Consistent with the results of previous experiments, BC275 almost completely suppressed tumor growth as long as antibody treatment was continuing while the other BsAbs had only a partial effect on leukemia growth (figure 6). Once antibody treatment was discontinued, tumors recurred in the BC275 groups, yet mice in the high-dose BC275 group had significantly smaller tumors compared with the high-dose BC133 ( $p=0.0002)$ or BC269 $(p=0.004)$ 
- BC275 + AML

- BC275

A

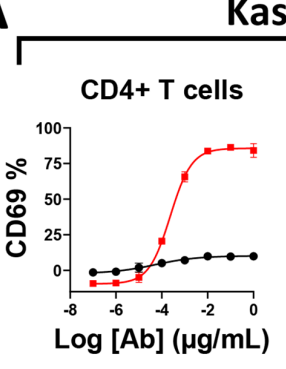

CD4+ T cells

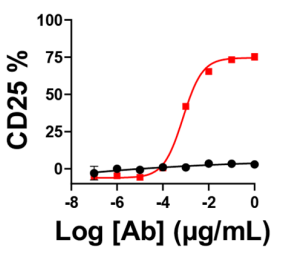

CD4+ $T$ cells

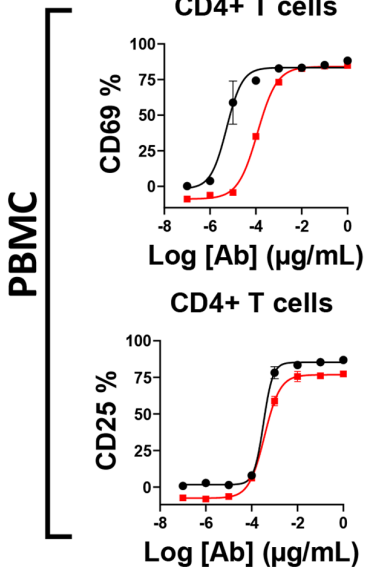

Kasumi-1

CD8+ T cells

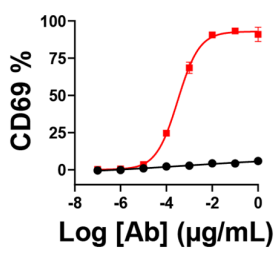

CD8+ T cells

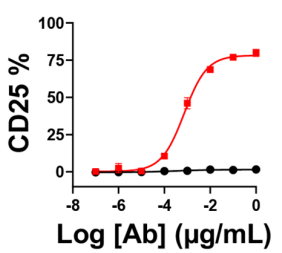

CD8+ T cells

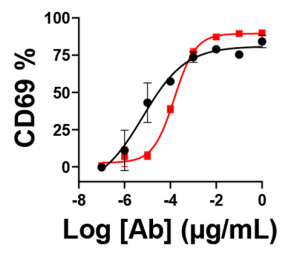

CD8+ T cells

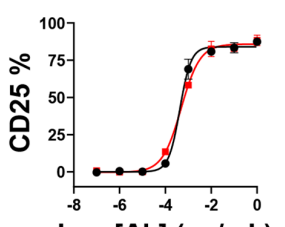

Log [Ab] $(\mu \mathrm{g} / \mathrm{mL})$
B

MOLM13

CD4+ T cells

CD8+ $\mathrm{T}$ cells

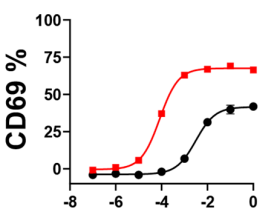

$\log [A b](\mu \mathrm{g} / \mathrm{mL})$

CD4+ T cells

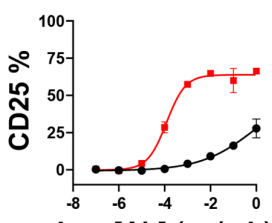

$\log [A b](\mu \mathrm{g} / \mathrm{mL})$

CD4+ T cells

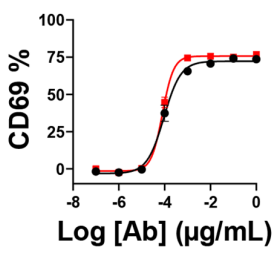

CD4+ T cells

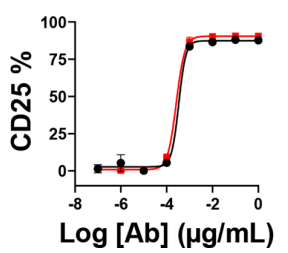

Figure 7 BC275 induces T cell activation in vitro. Freshly isolated T cells and peripheral blood mononuclear cells (PBMCs) were cultured without target cells or with CD33(+) acute myeloid leukemia (AML) lines Kasumi-1 (A) or MOLM13 (B) in the presence of dilutions of the CD33xCD3 bispecific antibodies (BsAbs) for 1 day or 4 days with an E:T ratio of 10. The expressions of CD69 (after 1 day) and CD25 (after 4 days) on CD4 and CD8 cells were assessed by flow cytometry. The percentage of positive cells is shown in the graphs.

groups. These data suggest that targeting the CD33 membrane IgC proximal domain with BC275 is superior to those $\mathrm{BsAb}$ against the $\mathrm{IgV}$ domain even when the affinity was inferior.

\section{BC275 activates T cells to proliferate and generate Th1 cytokines}

To further assess the in vitro pharmacology of BC275, non-activated naïve $\mathrm{T}$ cells or PBMCs were exposed to CD33(+) AML cell lines in the presence of BC275. BC275 did not activate $\mathrm{T}$ cells in the absence of target cells (figure 7). T cell activation markers were upregulated in the presence of AML cells or when BC275 was added to PBMCs, which contained autologous CD33expressing myeloid cells (figure 7 ). In addition to $\mathrm{T}$ cell activation, BC275 induced $\mathrm{T}$ cell proliferation in the presence of CD33(+) AML cells. Despite the ability to activate T cells to upregulate both early (CD69 after 1 day) and late (CD25 after 4 days) activation markers (figure 7), autologous monocytes within the PBMC population was poor in inducing $\mathrm{T}$ cell proliferation in the presence of BC275 (figure 8). When released cytokines were studied, isolated $\mathrm{T}$ cells did not generate cytokines when exposed to BC275 (figure 9A). Autologous myeloid cells showed poor induction of Th1 cytokine production, especially for IL-2, IL-10 and interferon- $\gamma$, while AML cells induced higher levels of cytokines in the presence of BC275 (figure 9B).

Although CD33 is a marker of the myeloid lineage, there is controversy around whether its expression on hematopoietic stem cells could subject them to being killed as a bystander. When CD34(+) HSPCs were stained with the BsAb, whereas BC275 stained all three AML cell lines, neither CD nor BM-derived CD34(+) cells reacted with the antibody (figure 9C). Based on these binding assays, BC275 should have preferential cytotoxicity against AML versus HSPCs. 


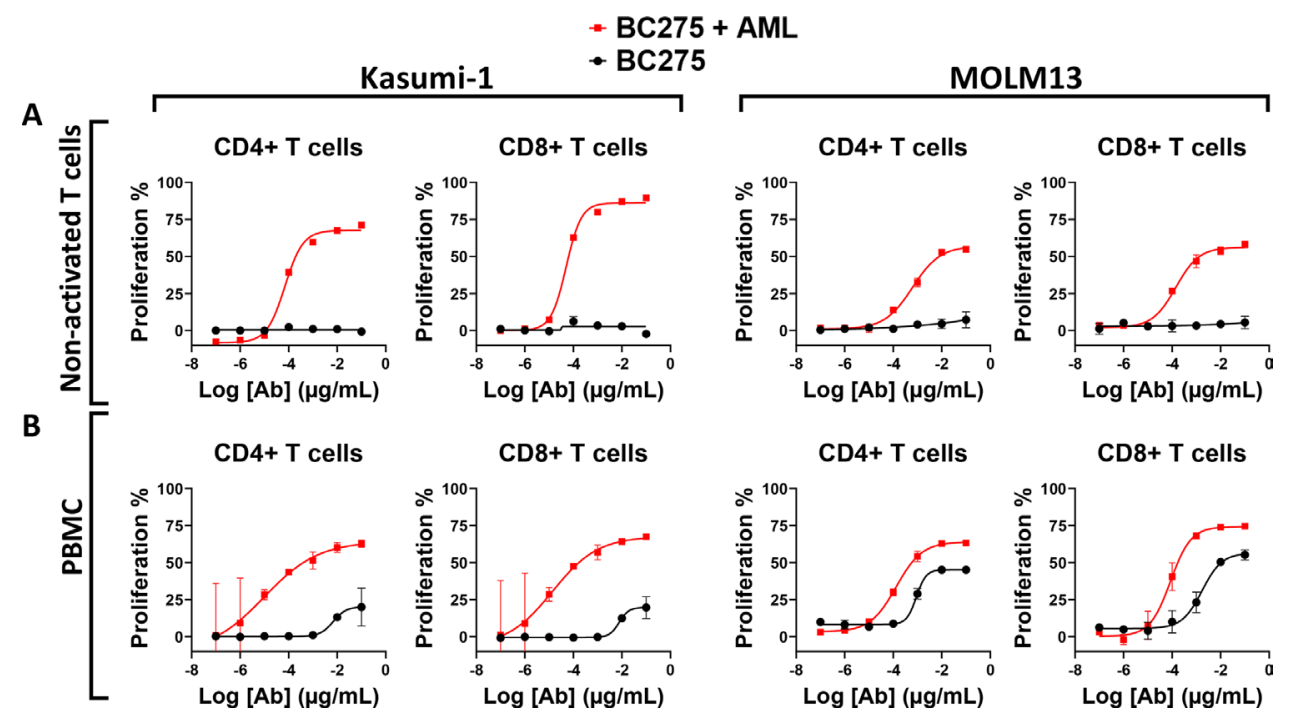

Figure 8 BC275 prompts T cell proliferation in vitro. Freshly isolated T cells (A) and peripheral blood mononuclear cells (PBMCs) (B) were labeled with carboxyfluorescein succinimidyl ester (CFSE) and cultured without target cells or with CD33(+) acute myeloid leukemia (AML) lines Kasumi-1 and MOLM13 in the presence of dilutions of the CD33xCD3 bispecific antibodies (BsAbs) for 4 days with an E:T ratio of 10. The percentage of CD4 and CD8 cells with dilutions of the CFSE dye was assessed by flow cytometry as proliferating cells.

\section{DISCUSSION}

Almost all the current anti-CD33 immunotherapies in clinical trials target the CD33 IgV domain. Recently, a monovalent $1+1 \mathrm{BsAb}$ targeting the IgC domain of CD33 was reported. ${ }^{9}$ Our BsAb differs in that (1) it uses the IgG-[L]-scfv $(2+2)$ format and binds bivalently to CD33 while functionally monovalent to CD3, a format shown to be superior when compared with alternative BsAb formats. ${ }^{8}$ In that report, we showed that the potency of the $2+2$ format in vivo is superior to other $2+2$ and $1+1$ bispecific constructs for GD2 and CD33, because of the optimal interdomain spacing and cis configuration of the binding domains. ${ }^{4} 8$ (2) The $2+2$ format versus the $1+1$ antibody $^{9}$ was more potent in T-cell-mediated cytotoxicity assays (low $\mathrm{pM}$ to $\mathrm{fM}$ vs high $\mathrm{pM}$ EC50) and T-cell activation
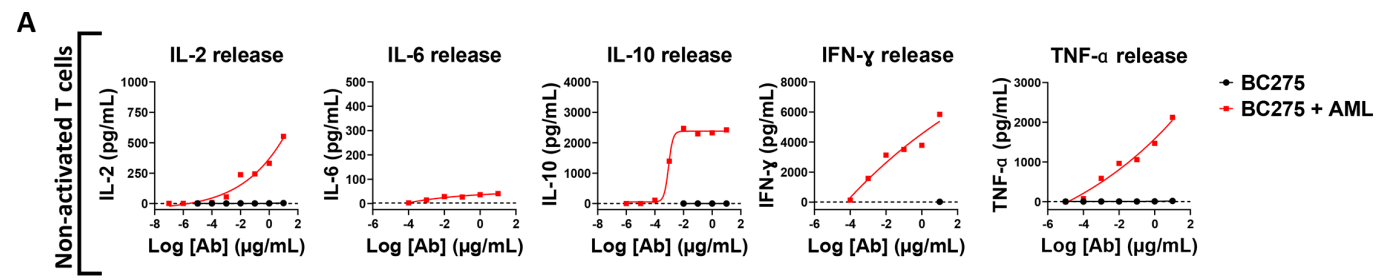

B
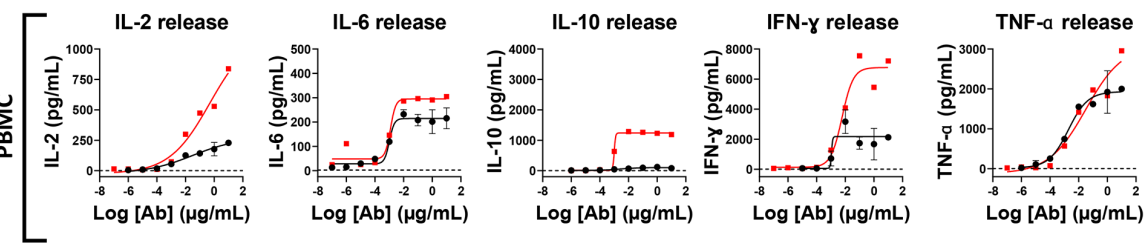

C
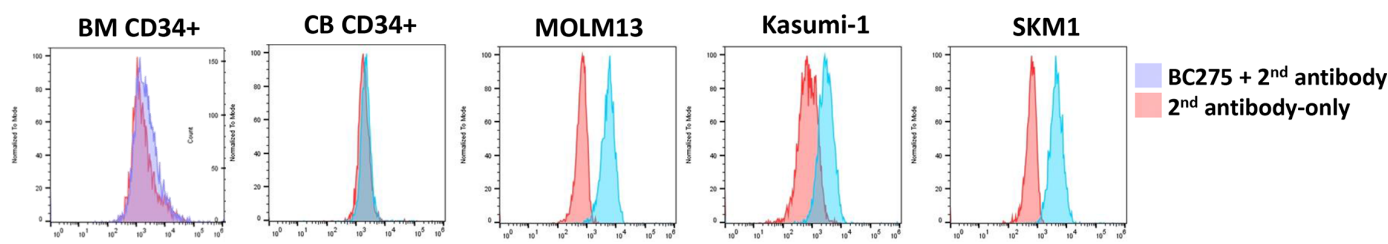

Figure 9 BC275 does not bind to hematopoietic stem and progenitor cells. Freshly isolated T cells (A) and peripheral blood mononuclear cells (PBMCs) (B) were cultured without target cells or with CD33(+) acute myeloid leukemia (AML) line Kasumi-1 in the presence of dilutions of the CD33xCD3 bispecific antibodies (BsAbs) for 1 day with an E:T ratio of 10. The production of Th1 cytokines was measured by a bead-based assay and flow cytometry. The binding of the BsAbs to bone marrow (BM) or cord blood (CB) CD34(+) hematopoietic stem and progenitor cells was assessed by flow cytometry (C). CD33(+) AML cell lines Kasumi-1, MOLM13, and SKM1 were used as positive controls. IFN- $\gamma$, interferon- $\gamma$; IL, interleukin; TNF- $\alpha$, tumor necrosis factor- $\alpha$. 
experiments (low pM to $\mathrm{fM}$ vs mid pM EC50). The higher potency of the $2+2$ format translates to superior anti-leukemic activity in vivo. Whereas our $2+2$ antibody required only 5 doses of $25 \mathrm{ng}$ to shrink the MOLM13 leukemia, the $1+1$ format required 13 doses of $1000 \mathrm{ng}$ to be effective against the same cell line. ${ }^{9}$ While a more direct comparison in the same preclinical models is the only valid way to compare potency, the choice of BsAb format remains a critical variable to be explored in future human trials.

GO, the only FDA-approved anti-CD33 agent, delivers the toxic ozogamicin payload to AML cells on internalization. However, pediatric patients with the heterozygous CT or homozygous TT rs12459419 SNP do not respond to GO. ${ }^{3}$ It was shown that these SNPs reduce the expression of the full-length CD33 that contains the IgV domain. ${ }^{3}$ Previously, we generated a $\mathrm{T}$ cell engaging BsAb against CD33 (BC133) by humanizing the clone M195 targeting the $\mathrm{IgV}$ domain. ${ }^{4}$ In this study, we reasoned that a BsAb against the membrane proximal IgC domain may be less affected by SNP variations. In addition, targeting membrane proximal domains has been shown to increase cytotoxicity for chimeric antigen receptors against CD22. ${ }^{10}$ This is the first systematic comparison of BsAbs targeting different domains of CD33 both in vitro and in animal models. In addition to BC133, we also tested a BsAb (BC269) using a rehumanized sequence of My96. Interestingly, binding of BC275, which is specific to the IgC domain, was lower in AML cells compared with the other BsAbs. In addition, BC275 was less potent in in vitro cytotoxicity assays. Nevertheless, BC275 consistently outperformed the other antibodies in vivo using different xenograft models of AML. It is unknown whether HIM3-4 monoclonal antibody or antibody-drug conjugates based on HIM3-4 would outperform monoclonal antibodies or antibody-drug conjugates based on other anti-CD33 antibodies.

It was previously reported that CD33 is expressed on activated T cells. ${ }^{71}$ The My96 clone (for building BC269) binds $64 \%$ of activated T cells, while HIM3-4 (for building BC275) binds only 14\% of activated $\mathrm{T}$ cells, ${ }^{7}$ which should translate into less $\mathrm{T}$ cell fratricide with $\mathrm{BC} 275$ when compared with BC269. It is possible that this could explain the superiority of BC275 over the other two BsAbs in vivo. Previous attempts to generate antibodies against the CD33 IgC domain have used IgC-only protein. However, the two antibodies that were generated failed to bind to the AML cells. ${ }^{12}$ It is possible that the resulting antibodies were recognizing epitopes on the CD33 IgC domain normally concealed by the $\mathrm{IgV}$ domain. In contrast, BC275 recognizes both the truncated CD33 IgC-only protein and the full-length CD33.

Currently, few BsAbs against CD33 are in clinical trials for treatment of relapsed/refractory AML. Many of these studies have not been encouraging partly because of the low response rates, although it should be noted that these early-phase trials are not necessarily designed to evaluate efficacy and may include patients treated with subtherapeutic dose levels. ${ }^{13}$ 14 Regardless, overcoming CD33 heterogeneity by engaging both the full length and the splice variant could enhance the BsAb approach. BC275 should be further explored as a potential candidate for clinical development in treating AML.

Contributors SSH conceived the study, performed the experiments, analyzed the data, designed the figures, and wrote the manuscript. MV and ME-C performed the experiments, analyzed the data, and edited the manuscript. HT, YF, and HFG performed the experiments and analyzed the data. HX performed SPR experiments. IC helped with SNP analysis of cell lines and edited the manuscript. N-KVC conceived the study, supervised the project, analyzed the data, and edited the manuscript.

Funding This work was supported in part by funds from Enid A. Haupt Endowed Chair, Kids Walk for Kids with Cancer NYC, Isabella Santos Foundation, Katie Find a Cure Foundation, the Robert Steel Foundation, Y-mAbs Therapeutics, Inc., and National Institutes of Health (CA232491), National Cancer Institute Cancer Center Support Grant P30 CA008748.

Competing interests SSH and N-KVC were named as co-inventors in patents on CD33 bispecific antibodies filed by Memorial Sloan Kettering Cancer Center (MSKCC). BC133 and BC275 were licensed to Y-mAbs Therapeutics by MSKCC. Both MSKCC and N-KVC have financial interest in Y-mAbs. SSH and MV are employees of $\mathrm{Y}$-mAbs Therapeutics. The remaining authors declare no competing financial interests.

Patient consent for publication Not required.

Provenance and peer review Not commissioned; externally peer reviewed.

Data availability statement All data contained within this manuscript are available on reasonable request to the corresponding author.

Open access This is an open access article distributed in accordance with the Creative Commons Attribution Non Commercial (CC BY-NC 4.0) license, which permits others to distribute, remix, adapt, build upon this work non-commercially, and license their derivative works on different terms, provided the original work is properly cited, appropriate credit is given, any changes made indicated, and the use is non-commercial. See http://creativecommons.org/licenses/by-nc/4.0/.

\section{ORCID iDs}

Madelyn Espinosa-Cotton http://orcid.org/0000-0002-8473-556X

Nai-Kong V Cheung http://orcid.org/0000-0001-6323-5171

\section{REFERENCES}

1 Kiem Hao T, Van Ha C, Huu Son N, et al. Long-Term outcome of childhood acute myeloid leukemia: a 10-year retrospective cohort study. Pediatr Rep 2020;12:8486.

2 Menzin J, Lang K, Earle CC, et al. The outcomes and costs of acute myeloid leukemia among the elderly. Arch Intern Med 2002;162:1597-603.

3 Lamba JK, Chauhan L, Shin M, et al. Cd33 splicing polymorphism determines Gemtuzumab Ozogamicin response in de novo acute myeloid leukemia: report from randomized phase III children's Oncology Group trial AAML0531. J Clin Oncol 2017;35:2674-82.

4 Hoseini SS, Guo H, Wu Z, et al. A potent tetravalent T-cell-engaging bispecific antibody against CD33 in acute myeloid leukemia. Blood Adv 2018;2:1250-8.

$5 \mathrm{Xu} \mathrm{H}$, Cheng M, Guo H, et al. Retargeting T cells to GD2 pentasaccharide on human tumors using bispecific humanized antibody. Cancer Immunol Res 2015;3:266-77.

6 Wang L, Hoseini SS, Xu H, et al. Silencing Fc domains in T cellEngaging bispecific antibodies improves T-cell trafficking and antitumor potency. Cancer Immunol Res 2019;7:2013-24.

7 Pérez-Oliva AB, Martínez-Esparza M, Vicente-Fernández JJ, et al. Epitope mapping, expression and post-translational modifications of two isoforms of CD33 (CD33M and CD33m) on lymphoid and myeloid human cells. Glycobiology 2011;21:757-70. 
8 Santich BH, Park JA, Tran H, et al. Interdomain spacing and spatial configuration drive the potency of IgG-[L]-scFv T cell bispecific antibodies. Sci Trans/ Med 2020;12. doi:10.1126/scitransImed. aax1315. [Epub ahead of print: 11 Mar 2020].

9 Nair-Gupta P, Diem M, Reeves D, et al. A novel C2 domain binding CD33xCD3 bispecific antibody with potent T-cell redirection activity against acute myeloid leukemia. Blood Adv 2020;4:906-19.

10 Haso W, Lee DW, Shah NN, et al. Anti-CD22-chimeric antigen receptors targeting $\mathrm{B}$-cell precursor acute lymphoblastic leukemia. Blood 2013;121:1165-74.

11 Hernández-Caselles T, Martínez-Esparza M, Pérez-Oliva AB, et al. A study of CD33 (SIGLEC-3) antigen expression and function on activated human T and NK cells: two isoforms of CD33 are generated by alternative splicing. J Leukoc Biol 2006;79:46-58.

12 Godwin CD, Laszlo GS, Wood BL, et al. The CD33 splice isoform lacking exon 2 as therapeutic target in human acute myeloid leukemia. Leukemia 2020;34:2479-83.

13 Ravandi F, Stein AS, Kantarjian HM. A phase 1 first-in-human study of AMG 330, an anti-CD33 bispecific T-cell engager (BiTE®) antibody construct, in relapsed/refractory acute myeloid leukemia (R/R AML). Blood 2018;132:25.

14 Westervelt P, Cortes JE, Altman JK. Phase 1 first-in-human trial of AMV564, a bivalent bispecific (2:2) CD33/CD3 T-cell Engager, in patients with relapsed/refractory acute myeloid leukemia (AML). Blood 2019;134:834. 